\title{
Activity and the Li abundances in the FGK dwarfs ${ }^{\star}, \star \star$
}

\author{
T. V. Mishenina ${ }^{1,2}$, C. Soubiran ${ }^{2}$, V. V. Kovtyukh ${ }^{1}$, M. M. Katsova ${ }^{3}$, and M. A. Livshits ${ }^{4}$ \\ 1 Astronomical Observatory, Odessa National University, and Isaac Newton Institute of Chile, Odessa Branch, \\ T.G. Shevchenko Park, 65014 Odessa, Ukraine \\ e-mail: tamar@deneb1.odessa.ua \\ 2 Université Bordeaux 1 - CNRS - Laboratoire d'Astrophysique de Bordeaux, UMR 5804, 33271 Floirac Cedex, France \\ e-mail: Caroline.Soubiran@obs.u-bordeaux 1.fr \\ 3 Sternberg State Astronomical Institute, Lomonosov Moscow State University, 13, Universitetsky Av., 119991 Moscow, Russia \\ e-mail: maria@sai.msu.ru \\ 4 Pushkov Institute of Terrestrial Magnetism, Ionosphere, and Radio Wave Propagation of Russian Academy of Sciences (IZMIRAN), \\ Troitsk, 142190 Moscow, Russia \\ e-mail: maliv@mail.ru
}

Received 7 November 2011 / Accepted 1 October 2012

\begin{abstract}
Aims. The aim of the present study is to determine the Li abundances for a large set of the FGK dwarfs and to analyse the connections between the Li content, stellar parameters, and activity.

Methods. The atmospheric parameters, rotational velocities and the $\mathrm{Li}$ abundances were determined from a homogeneous collection of the echelle spectra with high resolution and a high signal-to-noise ratio. The rotational velocities $v \sin i$ were determined by calibrating the cross-correlation function. The effective temperatures $T_{\text {eff }}$ were estimated by the line-depth ratio method. The surface gravities $\log g$ were computed by two methods: the iron ionization balance and the parallax. The LTE Li abundances were computed using synthetic spectra method. The behaviour of the $\mathrm{Li}$ abundance was examined in correlation with $T_{\mathrm{eff}},[\mathrm{Fe} / \mathrm{H}]$, as well as with $v \sin i$ and the level of activity in three stellar groups of the different temperature range.

Results. The stellar parameters and the Li abundances are presented for 150 slow rotating stars of the lower part of the main sequence. The studied stars show a decline in the Li abundance with decreasing temperature $T_{\text {eff }}$ and a significant spread, which should be due to the difference of age of stars. The correlations between the Li abundances, rotational velocities $v \sin i$, and the level of the chromospheric activity were discovered for the stars with $6000>T_{\text {eff }}>5700 \mathrm{~K}$, and it is tighter for the stars with $5700>T_{\text {eff }}>$ $5200 \mathrm{~K}$. The target stars with $T_{\text {eff }}<5200 \mathrm{~K}$ do not show any correlation between $\log A(\mathrm{Li})$ and $v \sin i$. The relationship between the chromospheric and coronal fluxes in active with detected $\mathrm{Li}$ as well as in less active stars gives a hint that there exist different conditions in the action of the dynamo mechanism in those stars.

Conclusions. We found that the Li-activity correlation is evident only in a restricted temperature range and the Li abundance spread seems to be present in a group of low chromospheric activity stars that also show a broad spread in the chromospheric vs. coronal activity.
\end{abstract}

Key words. stars: abundances - stars: activity - stars: late-type - stars: fundamental parameters - stars: rotation

\section{Introduction}

Li burns at temperatures of $2.5 \times 10^{6} \mathrm{~K}$ via $\alpha$ captures, which makes it a useful probe of the mixing in stars. The connection of the Li abundance with stellar activity has a long history (Herbig 1965; Pallavicini et al. 1987). It was soon realized that, like stellar activity, high $\mathrm{Li}$ abundance is found in young stars, although the Li abundance, by itself, is not sufficient to estimate the age of a star. Skumanich (1972) showed that both the chromospheric emission of the $\mathrm{Ca}$ II $\mathrm{H}$ and $\mathrm{K}$ lines and the rotational velocity decline as the square root of the age of stars (the Skumanich law). However, the Li abundance does not show a tight correlation with either (Duncan 1981; Duncan \& Jones 1983; Soderblom et al. 1993a). Whether a tight relation exists between the $\mathrm{Li}$ abundance and the stellar mass, the

* Based on the spectra collected with the ELODIE spectrograph using the 1.93-m telescope at the Observatoire de Haute Provence (CNRS, France).

$\star \star$ Full Table 3 is only available at the CDS via anonymous ftp to cdsarc.u-strasbg.fr $(130.79 .128 .5)$ or via

http://cdsarc.u-strasbg.fr/viz-bin/qcat?J/A+A/547/A106 spread, observed in the Li abundance owing to presence of spots on the star's surface (Soderblom et al. 1993a) or to the mainsequence (MS) depletion (Thorburn et al. 1993) is still an open question.

A spread of the Li abundance in the open cluster (OC) stars and binaries with the same mass and the Li depletion (Martin et al. 2002; King 2010), as well as higher Li abundance of rapidly rotating dwarfs (Cutispoto et al. 2003), are not corroborated by the theoretical predictions of the near-solar masses, in which the convection was only considered as a mixing mechanism (Randich 2010), and fast rotation intensifies the mixing process that leads to the Li destruction (e.g. Charbonnel et al. 1992).

The observation of the rotation and chromospheric emission in the F, G, and $\mathrm{K}$ dwarfs of the OC stars allowed a reliable agerotation relationship to be established that is one of the bases of the method of estimating age depending on the level of activity, so-called gyrochronology (Soderblom et al. 1993b,c; Barnes 2003). That paradigm can serve as a basis for understanding the relationship of activity, the light elements content and rotation (Barnes 2003). 
Analysis of the mechanisms that provided the Li overabundance was carried out for the stars of the RS CVn - type binaries with spots and a significant rate of rotation (Pallavicini et al. 1992; Pallavicini et al. 1993; Randich et al. 1993). In doing so, the moderate excess (overabundance) of Li compared to normal stars of the same spectral types was confirmed, and the correlation with the rotation parameter and the chromospheric fluxes was not discovered. The possibility of the Li enrichment, connected to the presence of spots and the production of additional $\mathrm{Li}$ in areas (Pallavicini et al. 1992), was not eliminated. The fresh isotopes of $\mathrm{Li}$ can be produced by the nuclear interactions of ions, accelerated at the surface of the flaring stars (see e.g. Canal et al. 1975; Livshits 1997; Tatischeff \& Thibaud 2007). Only one observational result that attests to the Li growth in the stellar area was obtained by Montes \& Ramsey (1998).

The chromospheric and coronal activities of late-type stars have been studied more in past decades (Guedel 2004; Guinnan \& Enge 2009; Katsova \& Livshits 2011). Katsova \& Livshits (2011) find that the ideas about the gyrochronology (Mamajek \& Hillenbrand 2008) is valid for the stars that are hotter than

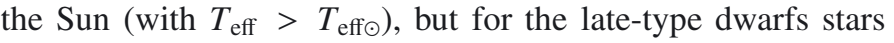
(with $T_{\text {eff }}<T_{\text {eff } \odot}$ ), whose convection zones are thicker than the solar ones, the activity evolves in time apparently according to the other law. Studing those stars that are both hotter and cooler than the Sun, can clarify the understanding of the features and causes of activity of the FGK dwarfs, as well as the real relation between the enhancement of Li and activity.

In this study we aim at finding some observational evidence of the Li abundance - activity behaviour in the stars in the lower part of the MS, that are the slow rotating dwarfs with masses close to the solar one.

The paper is divided as follows. The observational data are described in Sect. 2; Sect. 3 is devoted to the description of the levels of activity of the investigated stars; the methods and errors in determining of the rotational velocities, atmospheric parameters and the $\mathrm{Li}$ abundances are presented in Sects. 4-6, respectively. In Sect. 7, the Li abundance behaviour is considered in relation with the stellar and activity parameters. Conclusions are drawn in Sect. 8.

\section{Observations and the spectral processing}

The spectra of 150 stars were obtained using the $1.93 \mathrm{~m}$ telescope at the Observatoire de Haute-Provence (OHP, France), equipped with the échelle type spectrograph ELODIE (Barrane et al. 1996) which provides a resolving power of $R=42000$. Most of the target stars, 126 stars on the lower part of the MS that are examined in the present study, were taken from our previous paper Mishenina et al. (2008, M08). Some stars from M08 were eliminated from the analysis because it was impossible to estimate the $\mathrm{Li}$ abundances owing to distortions of the spectra in the $\mathrm{Li}$ line region. A set of 24 stars was added with spectra that were previously analysed by Mishenina et al. (2004) or retrieved from the ELODIE archive (Moultaka et al. 2004). All 150 spectra were processed homogeneously using the same methods.

The extraction of the 1D spectra and measurement of the radial velocities were performed with the standard on-line ELODIE reduction software, while the deblazing and removal of cosmic particles were carried out following Katz et al. (1998). Further processing of spectra (the continuous spectrum level set up and measurement of the equivalent widths) was conducted using the DECH20 software package (Galazutdinov 1992). Figure 1 shows the Li region in the spectra for some target stars.

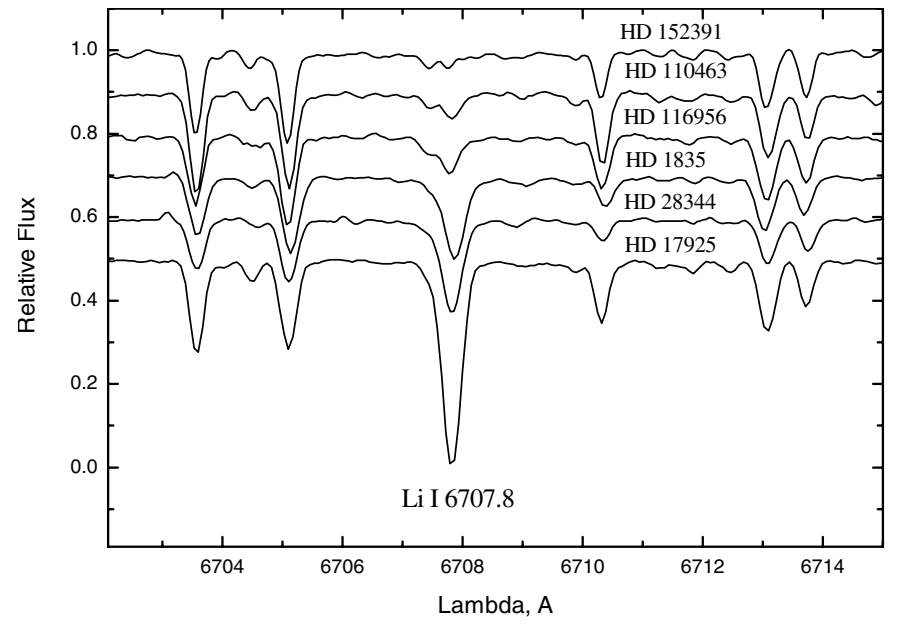

Fig. 1. The spectra in the Li $6707 \AA$ line region for some of the target stars.

Table 1. Comparison of the $\log R_{\mathrm{HK}}^{\prime}$ obtained by Wright et al. (2004) and from other sources.

\begin{tabular}{lccl}
\hline \hline$\Delta \log R_{\mathrm{HK}}^{\prime}$ & $\sigma$ & $N$ & Sources \\
\hline 0.00 & \pm 0.09 & 6 & Henry et al. (1996) \\
0.01 & \pm 0.04 & 7 & Baliunas et al. (1995) \\
-0.01 & \pm 0.07 & 7 & Hall et al. (2007) \\
\hline
\end{tabular}

\section{Levels of activity of the investigated stars}

In M08, we investigated the difference between the elemental abundances in active and inactive stars. We labelled as active those stars of the BY Dra type, RS CnV type, and flare Fl stars according to SIMBAD. We also classified six variable stars as active that showed evidence of the chromospheric activity in their $\mathrm{Ca}$ II and $\mathrm{H}_{\alpha}$ lines (see details in M08). In the present study, we also considered active those with a high level of activity, as discussed below.

As an indicator of the level of the chromospheric activity we examined the corresponding index $\log R_{\mathrm{HK}}^{\prime}$ that measures the chromospheric emission in the cores of the broad photospheric $\mathrm{Ca}$ II $\mathrm{H}$ and $\mathrm{K}$ absorption lines, normalized to the underlying photospheric spectrum. For a part of the target stars with $T_{\text {eff }}>$ $4800 \mathrm{~K}$, we found the $\log R_{\mathrm{HK}}^{\prime}$ values in Wright et al. (2004). We also used the data from the studies by Henri et al. (1996); Hall et al. (2007); Baliunas et al. (1995); and Maldonado et al.(2010). A total of 84 different stars were retrieved from those catalogues. The values of $\log R_{\mathrm{HK}}^{\prime}$ by Wright et al. (2004) are compared to those from different studies (Table 1), and agreement is good. For some stars where the $\log R_{\mathrm{HK}}^{\prime}$ index was not detected, we determined it on the base of the correlation between the chromospheric and the X-ray (Mamajek \& Hillenbrand 2008). The X-ray was taken from the ROSAT All-Sky Survey (Voges et al. 1999).

On having applied this index, it was necessary for us to pick out its value, corresponding to the "section boundary" between active and inactive stars. It should be noted that the values of indices themselves differ from each other in various studies. The discussion for some of these stars can be seen in the appendix.

We accepted that the transition between active and inactive stars occurs at $\log R_{\mathrm{HK}}^{\prime} \sim-4.75$. As indicated by Lovis et al. (2011) "this value corresponds to active stars according to the 
Table 2. Comparison of our $v \sin i$ values with those of other authors.

\begin{tabular}{rccl}
\hline \hline$\Delta v \sin i$ & $\sigma$ & $n$ & Source \\
\hline-0.15 & \pm 1.33 & 83 & Nordström et al. (2004) \\
-0.37 & \pm 1.18 & 67 & Valenti \& Fisher (2005) \\
-0.70 & \pm 2.00 & 26 & Tokovinin (1992) \\
-0.29 & \pm 0.71 & 25 & Fuhrmann (2008) \\
0.13 & \pm 0.96 & 14 & Benz \& Mayor (1984) \\
-0.56 & \pm 0.67 & 12 & Gaidos et al. (2000) \\
\hline
\end{tabular}

Notes. The mean difference and standard deviation are presented.

limit given by the Vaughan-Preston gap (Vaughan \& Preston 1980)". Moreover, a close value of $\log R_{\mathrm{HK}}^{\prime} \sim-4.80$ was declared in the study by Jenkins et al. (2011) and Katsova \& Livshits (2011).

\section{Rotational velocities}

We measured the rotational velocities $(v \sin i)$ of the target stars with a relation calibrated by Queloz et al. (1998), giving $v \sin i$ as a function of $\sigma \mathrm{RV}$, the standard deviation of the ELODIE crosscorrelation function, approximated by the Gaussian. The relation is the following:

$v \sin i=1.90 \sqrt{\sigma_{\mathrm{RV}}^{2}-\sigma_{0}^{2}}$.

The parameter $\sigma_{0}$ represents the mean intrinsic width for the non-rotating stars. It was calibrated by Queloz et al. (1998) as a function of $(B-V)$ :

$\sigma_{0}=0.27(B-V)^{2}+4.51$.

This $v \sin i$ calibration is valid for the stars in the colour range $0.7 \leq B-V \leq 1.4$, with slow and moderate rotation rates $\left(\leq 40 \mathrm{~km} \mathrm{~s}^{-1}\right)$ and the solar metallicities that are consistent with our stars.

We compared our values of $v \sin i$ with the results of determinations by other authors (Table 2). The comparison of our $v \sin i$ determinations with six other studies shows good compliance. The low values of $v \sin i$ are the upper limits of the projection of the rotational velocity definition. The obtained values of $v \sin i$ are given in Table 3.

\section{Atmospheric parameters}

We defined parameters $\left(T_{\text {eff }}, \log g,[\mathrm{Fe} / \mathrm{H}]\right)$ just for several newly included stars and for the others we used the determinations by Mishenina et al. (2004) and M08. The effective temperatures $T_{\text {eff }}$ were estimated by the line-depth ratio method (Kovtyukh et al. 2004). The surface gravity $\log g$ was computed by two methods: the iron ionization balance $\log g_{\mathrm{IE}}$ and the parallax $\log g_{\mathrm{P}}$. The results of applying of those two methods are in good compliance (M08). For cooler stars, which are short in the Fe II lines, the parallax was the only method used. The microturbulent velocity $V_{\mathrm{t}}$ was derived by considering that the iron abundance $\log A(\mathrm{Fe})$, obtained from the given $\mathrm{Fe}$ I line, is not correlated with the equivalent width $(\mathrm{EW})$ of that line. The adopted metallicity $[\mathrm{Fe} / \mathrm{H}]$ is the iron abundance, which was determined from the $\mathrm{Fe} \mathrm{I}$ lines.

The comparison of the obtained atmospheric parameters with those by the other authors is given in Table 4. As is evident, there is no significant difference between various determinations. The errors of the obtained temperature determinations are given for each star, once determined by the line-depth ratio method. For $\log g, V_{\mathrm{t}}$, and $[\mathrm{Fe} / \mathrm{H}]$, we estimated the errors to be 0.2 dex, 0.2 dex, and 0.05 dex, respectively.

\section{Determination of the $\mathrm{Li}$ abundance}

We used the grid of the stellar atmosphere models under the overshooting approximation from Kurucz (1993) to compute the abundances of $\mathrm{Li}$ and metallicity $[\mathrm{Fe} / \mathrm{H}]$. The $\mathrm{Li}$ abundances in the investigated stars were obtained by fitting the observational profiles to the synthetic spectra that were computed by the STARSP LTE spectral synthesis code, developed by Tsymbal (1996). Considering the wide temperature and metallicity ranges of the target stars, we made every effort to compile the full list of the atomic and molecular lines close to the ${ }^{7} \mathrm{Li} 6707 \AA$ line (Mishenina \& Tsymbal 1997). Calculating the synthetic spectra, we used the values of vsini, obtained with the relation calibrated by Queloz et al. (1998) described above. The stellar parameters and the Li abundances, measured in the present study, as well as the basic stellar characteristics are presented in Table 3.

Table 5 shows the example of three stars with different parameters: the error determination for some typical models of the investigated stars, for strong lines with $E W_{1}=100 \mathrm{~m} \AA$ and for weak lines with $E W_{2}=5 \mathrm{m \AA}$ with $\Delta T_{\text {eff }}=+100 \mathrm{~K}$ (Col. 1); $\Delta \log g=-0.2$ (Col. 2); $\Delta V_{\mathrm{t}}=+0.2 \mathrm{~km} \mathrm{~s}^{-1}$ (Col. 3); $\Delta E W_{1}= \pm 3 \mathrm{~m} \AA$ (Col. 4); and $\Delta E W_{1}= \pm 2 \mathrm{~m} \AA$ (Col. 5). The total error is given in Col. 6. As seen in Table 5, the total uncertainty grows for the stars with decreasing temperatures and depends on the EW lines. It reaches $0.10-0.14$ dex in the abundance determination for strong lines (near $100 \mathrm{m \AA}$ ) and 0.18 0.20 dex for weak lines (near $5 \mathrm{~m} \AA$ ).

The comparison of the stellar parameters and the Li abundance with the results by other authors is given in Table 6 . In Table 7 we compared our $\mathrm{Li}$ abundances with those in some papers, which have only one common star with our work. The parameters and the Li abundance, determined by us, are mainly in good compliance with the determinations of other authors. The comparison was made for the precise determinations of the Li content, but not for the estimations of the upper limits of the $\mathrm{Li}$ abundance. As marked in the study by Lubin et al. (2010), which collected the determinations of the Li abundance, made in thirty studies by different authors and for various objects, for $\log A(\mathrm{Li})>1.5$ there is a relatively small dispersion in the Li abundance detections among various measurement programmes, while the scattering increases for lower $\log A(\mathrm{Li})$. The difference in $\log A(\mathrm{Li})$ is about $0.5-1$ dex.

\section{Lithium, stellar parameters, and activity}

Our target stars have been selected as the stars belonging to the lower part of the MS upon photometric criterion $\left(M_{v}-(B-V)\right)$, where $M_{v}=V+5+2.5 \log \pi(\mathrm{M} 08)$. Figure 2 shows the positions of the investigated stars at the luminosity $\log L / L_{\odot}$ against the effective temperature $\log T_{\text {eff }}$ diagram where $\log L / L_{\odot}=$ $0.4\left(4.75-M_{\mathrm{bol}}\right)$, and $M_{\mathrm{bol}}=M_{v}+\mathrm{BC}$ (the bolometric corrections BC were taken from Flower 1996) and the evolutionary tracks by Girardi et al. 2000. Those stars are the FGK spectral type ones, and as can be seen from Fig. 2, they are almost evenly distributed over the effective temperature and have masses of about the solar one (from 0.7 to $1.1 M_{\odot}$ ). They have the average value of gravity $\langle\log g\rangle$ equal to $4.45 \pm 0.16$ and metallicity $\langle[\mathrm{Fe} / \mathrm{H}]\rangle=-0.01 \pm 0.17$. The rotational velocities of the 
Table 3. Stellar parameters, determined in the present study.

\begin{tabular}{lccccccccccc}
\hline \hline $\mathrm{HD}$ & $T_{\mathrm{eff}}$ & $\log g(1)$ & $\log g(2)$ & {$[\mathrm{Fe} / \mathrm{H}]$} & $v \sin i$ & Object type & $R_{\mathrm{x}}$ & $\log R_{\mathrm{HK}}^{\prime}$ & $\operatorname{Ref}$ & $\log A(\mathrm{Li})$ & Class \\
\hline 166 & 5514 & 4.6 & 4.6 & 0.16 & 4.0 & $\mathrm{BY}$ & -4.31 & -4.33 & & 2.24 & $\mathrm{~A}$ \\
1835 & 5790 & 4.5 & $\ldots$ & 0.13 & 6.51 & $\mathrm{BY}$ & -4.58 & -4.44 & $\mathrm{~W} 04$ & 2.45 & $\mathrm{~A}$ \\
3651 & 5277 & 4.5 & 4.5 & 0.15 & 0.0 & $\mathrm{~V}$ & -6.07 & -5.02 & $\mathrm{~W} 04$ & $<-0.52$ & $\mathrm{wA}$ \\
4256 & 5020 & 4.3 & 4.3 & 0.08 & 1.5 & $\ldots$ & $\ldots$ & $\ldots$ & & $<-0.40$ & $\ldots$ \\
4628 & 4905 & 4.6 & $\ldots$ & -0.21 & 1.5 & $\ldots$ & -5.93 & -4.85 & $\mathrm{H} 96, \mathrm{~B} 95$ & $<-0.45$ & $\mathrm{wA}$ \\
4635 & 5103 & 4.4 & 4.4 & 0.07 & 1.4 & $\ldots$ & $\ldots$ & -4.67 & $\mathrm{~W} 04$ & $<0.15$ & $\mathrm{~A}$ \\
4913 & 4342 & 4.4 & $\ldots$ & 0.05 & 2.15 & $\ldots$ & $\ldots$ & $\ldots$ & & $<-0.90$ & $\ldots$ \\
6660 & 4759 & 4.6 & $\ldots$ & 0.08 & 2.5 & $\ldots$ & $\ldots$ & $\ldots$ & & $<-0.50$ & $\ldots$ \\
7590 & 5962 & 4.4 & 4.4 & -0.10 & 6.7 & $\mathrm{BY}$ & -4.69 & -4.53 & $\mathrm{~W} 04$ & 2.73 & $\mathrm{~A}$ \\
7924 & 5165 & 4.4 & 4.4 & -0.22 & 1.1 & $\ldots$ & -5.71 & -4.83 & $\mathrm{~W} 04$ & $<-0.30$ & wA \\
$\ldots$ & $\ldots$ & $\ldots$ & $\ldots$ & $\ldots$ & $\ldots$ & $\ldots$ & $\ldots$ & $\ldots$ & $\ldots$ & $\ldots$ & $\ldots$ \\
\hline
\end{tabular}

Notes. $\log g(1)$ : spectroscopic, $\log g(2)$ : astrometric, object type from SIMBAD, Rx adopted from the ROSAT catalogues or estimated from the ROSAT archive data, indices $\log R_{\mathrm{HK}}^{\prime}$ retrieved from different sources, the measured Li abundance, and the class of activity. The log $R_{\mathrm{HK}}^{\prime}$ values, taken from Wright et al. (2004) are marked by W04; from Henri et al. (1996) - marked by H96; from Hall et al. (2007) - by H07; from Baliunas et al. (1995) - by B95, from Maldonado et al. (2010) - by M10. The stars with weak levels of the solar-type activity are marked by wA; the stars with high levels of the solar-type activity are marked by A in the last column.

Table 4. Comparison of atmospheric parameters obtained by us with those by other authors.

\begin{tabular}{rcccl}
\hline \hline$\Delta T_{\text {eff }}$ & $\Delta \log g$ & $\Delta[\mathrm{Fe} / \mathrm{H}]$ & $n$ & Source \\
\hline $16 \pm 68$ & & & 32 & Masana et al. (2006) \\
$-8 \pm 63$ & $-0.14 \pm 0.18$ & $-0.03 \pm 0.08$ & 67 & Valenti \& Fisher (2005) \\
$42 \pm 36$ & $-0.07 \pm 0.15$ & $0.00 \pm 0.05$ & 26 & Fuhrmann (2008) \\
$1 \pm 73$ & $-0.01 \pm 0.17$ & $-0.03 \pm 0.04$ & 14 & Gaidos \& Gonzalez (2002) \\
\hline
\end{tabular}

Table 5. Influence of stellar parameters on Li abundance determination.

\begin{tabular}{|c|c|c|c|c|c|c|}
\hline \multicolumn{5}{|c|}{ Parameter deviations } & \multicolumn{2}{|c|}{ Total error } \\
\hline $\begin{array}{l}\Delta T_{\text {eff }} \\
-100 \mathrm{~K}\end{array}$ & $\begin{array}{l}\Delta \log g \\
-0.2\end{array}$ & $\begin{array}{r}\Delta V_{\mathrm{t}} \\
+0.2\end{array}$ & $\begin{array}{c}E W=100 \mathrm{~m} \AA \\
+3 \mathrm{~m} \AA\end{array}$ & $\begin{array}{l}E W=5 \mathrm{~m} \AA \\
\quad+2 \mathrm{~m} \AA\end{array}$ & strong lines & weak lines \\
\hline \multicolumn{7}{|c|}{$T_{\text {eff }}=5800, \log g=4.5, V_{\mathrm{t}}=1.0 \mathrm{~km} \mathrm{~s}^{-1},[\mathrm{Fe} / \mathrm{H}]=0.0$} \\
\hline \multicolumn{7}{|c|}{$T_{\text {eff }}=5000, \log g=4.5, V_{\mathrm{t}}=1.0 \mathrm{~km} \mathrm{~s}^{-1},[\mathrm{Fe} / \mathrm{H}]=0.0$} \\
\hline \multicolumn{7}{|c|}{$T_{\text {eff }}=4600, \log g=4.5, V_{\mathrm{t}}=1.0 \mathrm{~km} \mathrm{~s}^{-1},[\mathrm{Fe} / \mathrm{H}]=0.0$} \\
\hline
\end{tabular}

Table 6. Comparison of atmospheric parameters and Li abundance with those obtained by other authors.

\begin{tabular}{lccccl}
\hline \hline$\Delta T_{\text {eff }}$ & $\Delta \log g$ & $\Delta[\mathrm{Fe} / \mathrm{H}]$ & $A(\mathrm{Li})$ & $n$ & Source \\
\hline $67 \pm 137$ & $0.23 \pm 0.11$ & $0.11 \pm 0.08$ & $-0.01 \pm 0.10$ & 3 & Chen et al. (2001) \\
$76 \pm 69$ & & & $0.09 \pm 0.14$ & 3 & Favata et al. (1997) \\
$29 \pm 67$ & $0.06 \pm 0.17$ & $0.03 \pm 0.06$ & $-0.08 \pm 0.12$ & 10 & Takeda et al. (2005, 2007) \\
$24 \pm 51$ & $0.11 \pm 0.15$ & $0.04 \pm 0.09$ & $0.00 \pm 0.04$ & 5 & Luck \& Heiter $(2006)$ \\
\hline
\end{tabular}

investigated stars generally do not exceed $6 \mathrm{~km} \mathrm{~s}^{-1}$ (Fig. 3, upper panel). The X-ray flux distribution has just one peak and the average value equals $-4.58 \pm 0.58$; however, the distribution of the intensities of the $\mathrm{H}$ and $\mathrm{K}$ Ca II emission lines in the chromospheres has two peaks that are separated by value $\log R_{\mathrm{HK}}^{\prime}=-4.75$ (Fig. 3, bottom panel), which correspond to the values adopted by us (Sect. 3). Comparison of this histogram with similar distributions for active late-type stars (see, for instance, Katsova \& Livshits 2011) shows that our set of stars is characterized by relatively moref stars with the higher activity.

The $\mathrm{Li}$ is detected in 43 stars among 69 stars with a high level of the chromospheric activity, while it is detected in ten stars among 31 dwarfs with weak level of the solar-type activity, i.e. in about $62 \%$ and $31 \%$, respectively. We have confirmed our previous result (M08) that the frequency of stars with the observed $\mathrm{Li}$ abundance is higher in active stars.

\subsection{Lithium abundance behaviour in relation to $T_{\text {eff }}$ and metallicity}

The obtained $\mathrm{Li}$ abundances $\log A(\mathrm{Li})$ are presented as a function of the effective temperature $T_{\text {eff }}$ in Fig. 4. Our set of stars showed a typical decrease of the $\mathrm{Li}$ abundance with decreasing temperature as predicted by the theoretical calculations (e.g. 
Table 7. Comparison of the parameters and the Li abundance for some stars

\begin{tabular}{lcccl}
\hline \hline HD & $T_{\text {eff }}$ & $\log g$ & $\log A(\mathrm{Li})$ & \\
\hline \multirow{2}{*}{17925} & 5225 & 4.56 & 2.68 & this work \\
& 5235 & 4.66 & 2.82 & Takeda et al. (2005) \\
& 5012 & 4.60 & 2.45 & Christian et al. (2005) \\
30495 & 5820 & 4.40 & 2.35 & this work \\
& 5880 & 4.67 & 2.45 & Israelian et al. (2004) \\
190406 & 5905 & 4.30 & 2.28 & this work \\
& 5797 & 4.38 & 2.26 & Chen \& Zhao (2006) \\
219623 & 5949 & 4.20 & 2.60 & this work \\
& 6103 & 4.18 & 2.76 & Takeda et al. (2005) \\
& 6130 & 4.21 & 2.68 & Lambert et al. (1991) \\
\hline
\end{tabular}

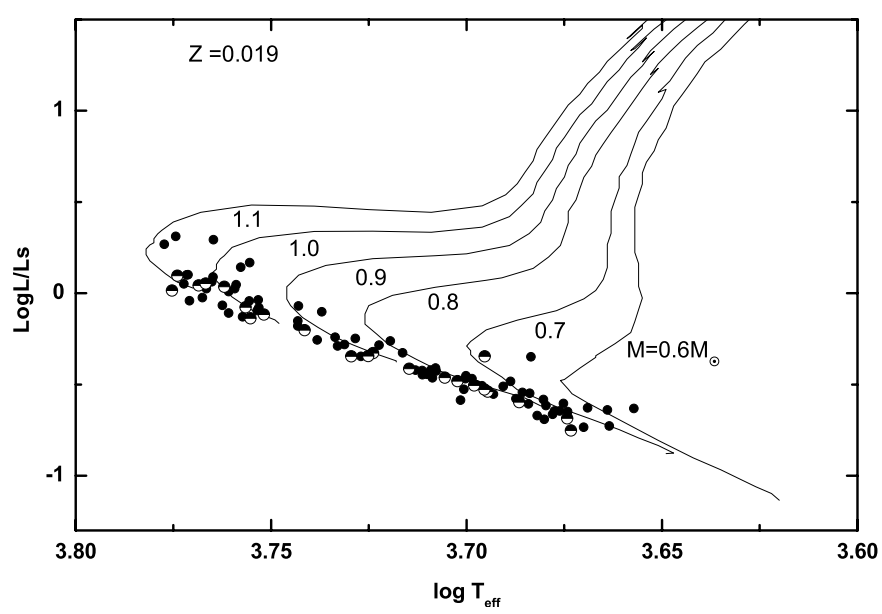

Fig. 2. Position of our target stars in the $\log L / L_{\odot}$ vs. $\log T_{\text {eff }}$ diagram. Evolutionary tracks were taken from Girardi et al. (2000), the stars of the BY Dra types are marked as semi-full circles, the other stars as full circles.

D’Antona \& Mazzitelli 1997), but with a rather wide spread. The active stars have higher values for the Li abundance than the usual dwarfs at any given temperature.

The excess of Li may be explained, in particular, by the production of additional $\mathrm{Li}$ in the flares. To refine that assumption, it is necessary to study the isotopic ratio ${ }^{6} \mathrm{Li} /{ }^{7} \mathrm{Li}$, which requires spectra with resolution better than 100000 and a signal-to-noise ratio greater than 400 . That is beyond the scope of the present study.

This spread is unlikely owing to the difference in metallicity, because Fig. 5 with $\log A(\mathrm{Li})$ vs. $[\mathrm{Fe} / \mathrm{H}]$ shows evidence of the fairly compact distribution of metallicity around the solar value for active stars.

Then, we compared the Li abundance behaviour with "the middle course of the Li trend" for several OCs of different ages (in Fig. 3 the trends are given schematically). We can see in Fig. 3, the Li abundance spread is due to different ages of stars. Younger stars have higher $\mathrm{Li}$ abundance, but, at the same time, they are actually active stars.

\subsection{Relationship between the Li abundance, rotation, and the chromospheric activity index}

Below we consider the dependencies of the Li abundance on other parameters, including the activity indices, for the stars of three different temperature ranges: the first group with
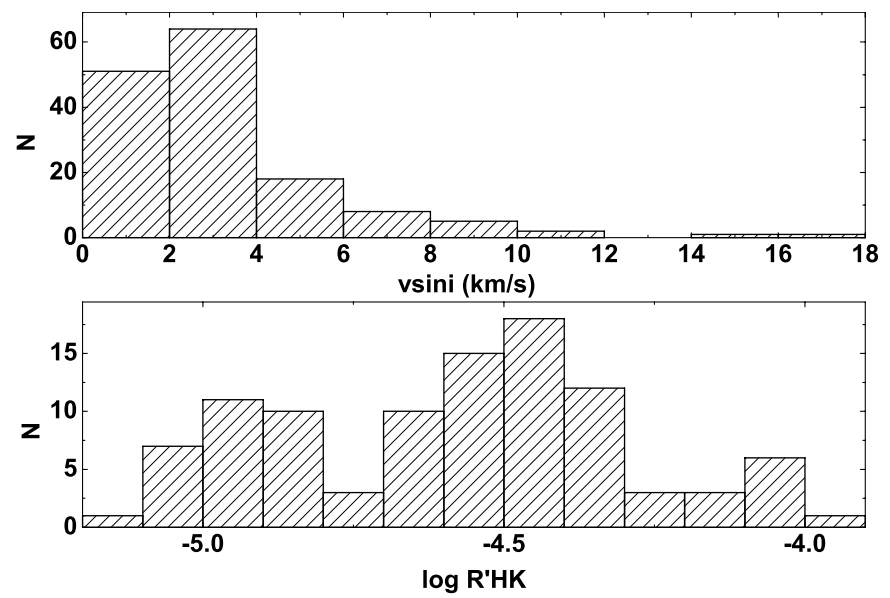

Fig. 3. The distribution of $v \sin i$ and $\log R_{\mathrm{HK}}^{\prime}$ of our target stars.

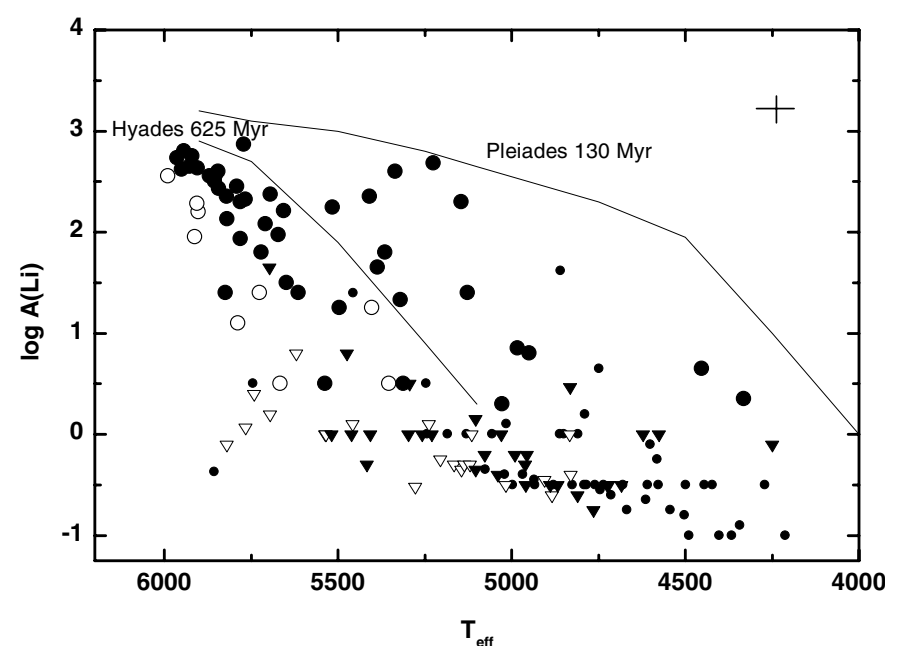

Fig. 4. Lithium abundances vs. $T_{\text {eff }}$. The medium trend of the Pleiades and the Hyades is represented according to Soderblom et al. (1993a) and Thorburn et al. (1993), respectively. The stars with a high level of the chromospheric activity $\left(\log R_{\mathrm{HK}}^{\prime}>-4.75\right)$ are marked as full circles, those with a weak level of activity $\left(\log R_{\mathrm{HK}}^{\prime}<-4.75\right)$ as open circles. Triangles denote the upper limits of the Li determinations. The stars, for which $\log R_{\mathrm{HK}}^{\prime}$ are not defined, are marked as small full circles.

$6000>T_{\text {eff }}>5700$, the second group with $5700>T_{\text {eff }}>5200$, and the third group with $T_{\text {eff }}<5200 \mathrm{~K}$.

Those dependencies are quite clearly expressed for the first two groups of stars: both the Li abundance and the chromospheric activity grow for the faster rotators. The values of $\log A(\mathrm{Li})$ are higher for the solar-type stars than for the stars cooler than the Sun (i.e. the late-type $G$ stars). The spread of these parameters in Figs. 6a, b and 7a, b is somewhat greater for the first group of stars than for the second one.

Those results can be seen more evidently we compare the $\mathrm{Li}$ abundance with the chromospheric activity (Fig. 8). It is also clear that the $\log A(\mathrm{Li})$ values are greater for the stars in the first group than in the second one. The correlation coefficient is 0.64 for the solar-type stars, and 0.77 for the stars cooler than the Sun. The spread of values of $\log A(\mathrm{Li})$ is less for the stars of the second group

For a few stars with $T_{\text {eff }}$ lower than $5200 \mathrm{~K}$, a detectable value of the Li abundance was obtained. Those stars (see Fig. 6c) demonstrate the absence of the correlation practically between $\log A(\mathrm{Li})$ and $v \sin i$ and there are some outliers with high 


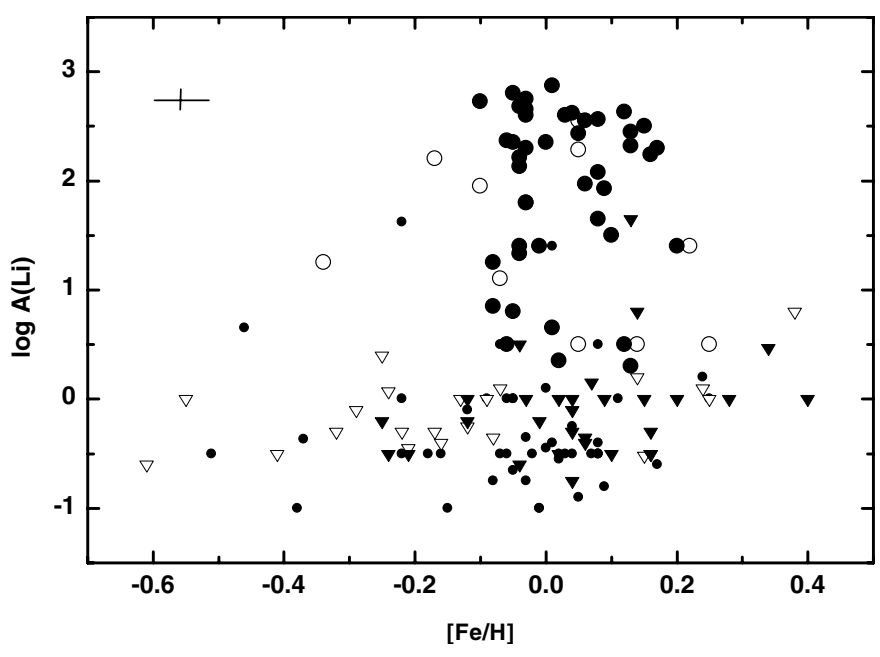

Fig. 5. The $\mathrm{Li}$ abundances vs. $[\mathrm{Fe} / \mathrm{H}]$, the notation is the same as in Fig. 4.

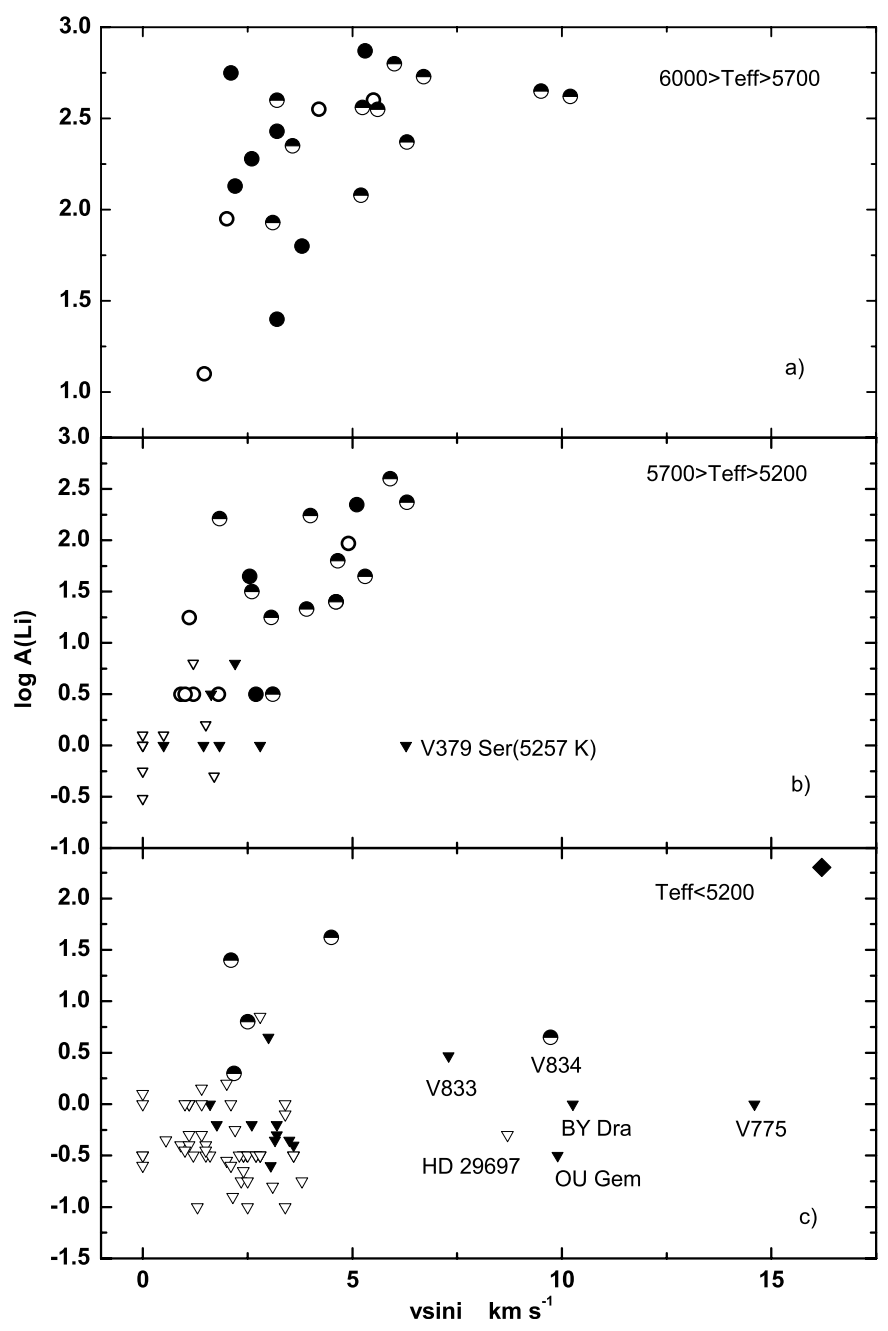

Fig. 6. Dependence of the Li abundance on $v \sin i$.

velocities and low Li abundances. Those stars, except HD 29697 , are known stars of the BY Dra type (namely, V833 Tau, V834 Tau, BY Dra, OU Gem, and V775 Her) binaries, stars with low temperatures and very weak lines of $\mathrm{Li}$ in the spectra of the main component.

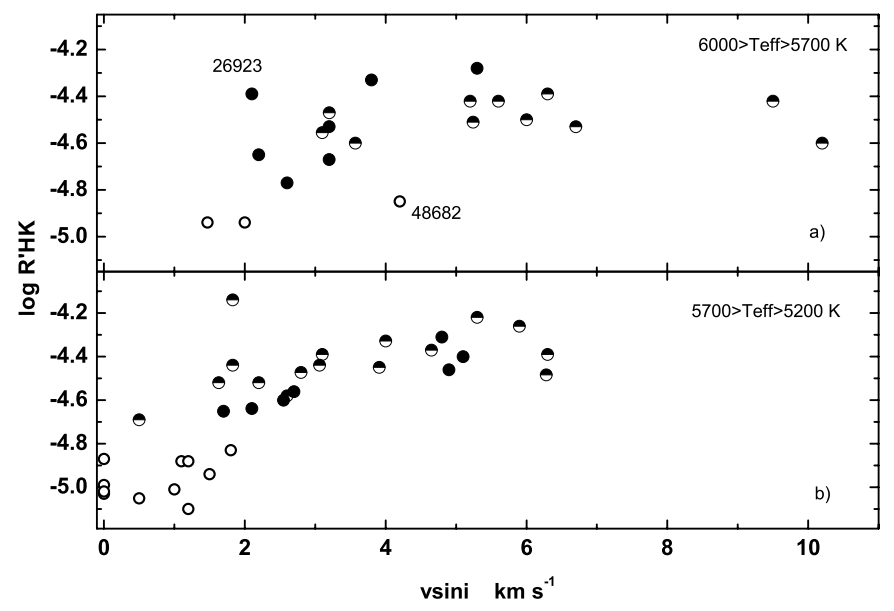

Fig. 7. Dependence of the $\log R_{\mathrm{HK}}^{\prime}$ on $v \sin i$.

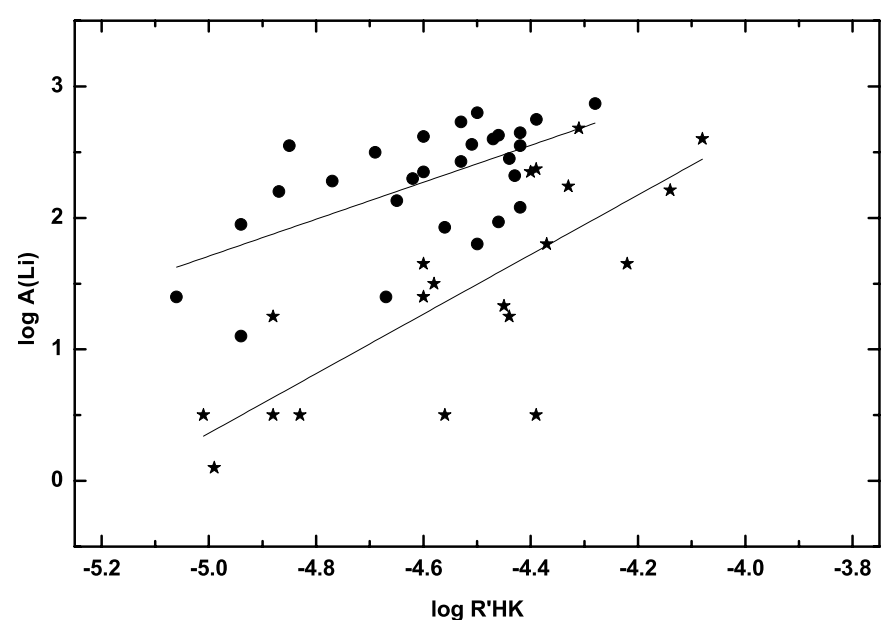

Fig. 8. Dependence of the Li abundance on $\log R_{\mathrm{HK}}^{\prime}$ for the stars with $6000>T_{\text {eff }}>5700$ (full circles) and with $T_{\text {eff }}$ from 5700 to $5200 \mathrm{~K}$ (asterisks).

The lower abundances of $\mathrm{Li}$ for the later G stars are associated with the fact that the convection zones of those stars become deeper than that on the Sun. The decrease in the Li abundance must be observed at $T_{\text {eff }}<5800 \mathrm{~K}$ where the bottom of the surface convection zone reached the regions where $\mathrm{Li}, \mathrm{Be}$, and B burn (e.g. Michaud et al. 2004) and the strong convection destroys Li at $T_{\text {eff }}<5400 \mathrm{~K}$ (e.g. Iben 1965; D'Antona \& Mazzitelli 1984, etc.).

Good correlations between $\log A(\mathrm{Li})$ and $\log R_{\mathrm{HK}}^{\prime}$ with $v \sin i$ for G-type stars are evidence that the rotation is a key factor in the physical processes that are responsible for the levels of the $\mathrm{Li}$ abundance and the chromospheric activity. Some distinctions of those dependences are due to the different role of the rotation rate in both processes. The $\mathrm{Li}$ abundance depends on the axial rotation rate indirectly through the age, while the dynamo process is a direct consequence of interaction between the rotation and the turbulent convection.

\subsection{How can studying lithium help with understanding the general problems of stellar activity?}

Up to now stellar activity has been studied for the young objects with activity close to saturation and less active stars observed during execution of exoplanet search programmes (for example, Wright et al. 2004; Martínez-Arnáiz et al. 2011). Previously, we 


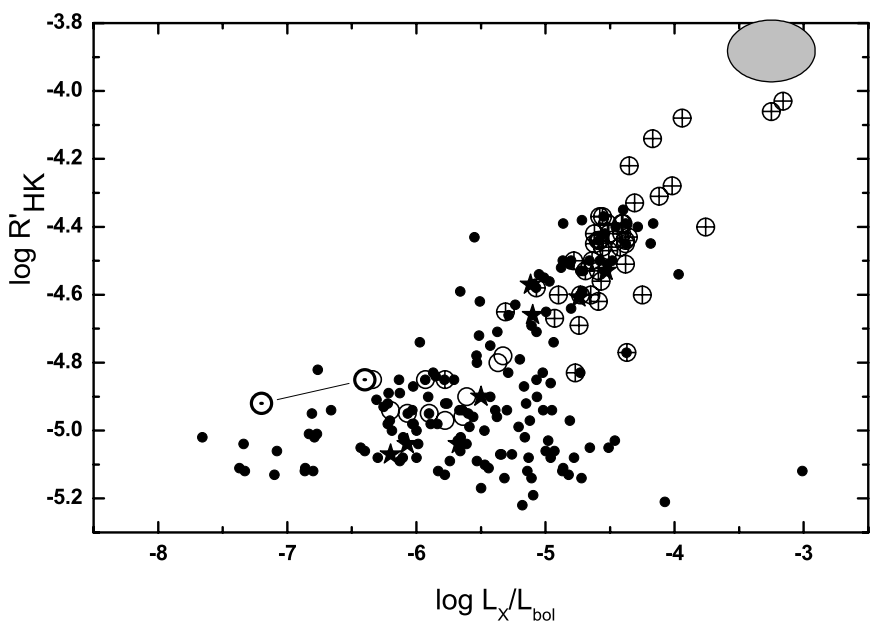

Fig. 9. Diagram of the chromospheric and coronal activity for the latetype stars. The stars of the basic data set are marked as dots. The corresponding references are given above, or the summary table see Katsova \& Livshits (2011). The investigated stars with measured Li abundance and with direct measurements of the $\log R_{\mathrm{HK}}^{\prime}$ indices (from the studies mentioned above) are marked as crosses inside the circles. According to the type of a cycle, the stars of group "Excellent" are marked as circles, the stars of "Good" group are indicated as asterisks; the Sun at the maximum and at the minimum is denoted by its own sign and connected by the direct line.

studied the relationship between the coronal and chromospheric activity (Katsova \& Livshits 2006, 2011). Here we also consider a set of stars that contains objects with a detectable Li line and with moderate activity levels. Data on the chromospheric activity indices are adopted from the papers cited in Sect. 3 . The soft $\mathrm{X}$-ray radiation of all those stars was adopted from the ROSAT data (Schmitt \& Liefke 2004; Huensch et al. 1999) and the XMM-Newton observations (Poppenhaeger et al. 2010, 2011). The data for more than 50 stars with detected $\mathrm{Li}$ abundances allows us to fill up a gap between low- and saturated activity on the chromosphere-corona diagram constructed by Katsova \& Livshits (2011) for 172 stars including the Sun. In this way, the whole set of stars includes stars with the low- and high activity levels.

The results are presented in Fig. 9. Newly added stars with detected Li hardly deviate from the straight line by Mamajek \& Hillenbrand (2008) corresponding to the linear regression between $\log R_{\mathrm{HK}}^{\prime}$ and $\log L_{\mathrm{X}} / L_{\mathrm{bol}}$. Most stars of that group are hotter than the Sun. Since the activity level is associated with the age, displacement along this line describes an evolution in the activity of such stars. The activity of all late-type stars evolves quickly during the first 1-2 Gyr, then their paths diverge and the stars cooler than the Sun displace below this line. Only several stars with detected $\mathrm{Li}$ are among these objects, while the others, revealed earlier on the same kind of diagram by Katsova \& Livshits (2011) are less active.

Physically, the change in the relationship between the chromospheric and coronal indices reflects the change in the properties of the activity. Probably, that can be due to various depths of the convection zone in those stars and the change in conditions for the dynamo mechanism. A broad spread in estimations of the ages of late $\mathrm{G}$ and $\mathrm{K}$ stars from their activity levels and from the $\mathrm{Li}$ abundance is due to that circumstance. We believe that Li may be a better indicator of the age than the activity. We point out that the stars, classified in the HK Project as stars with "Excellent" cycles, are located on the diagram outside the main group of the $\mathrm{F}$ and earlier G-stars because the formation processes of the stellar cycles can be related to the above problems.

\section{Conclusion}

The physical parameters, $[\mathrm{Fe} / \mathrm{H}]$, and the $\mathrm{Li}$ abundances were determined homogeneously for a sample of 24 FGK dwarfs, and the behaviour of the $\mathrm{Li}$ abundance was analysed for 150 stars. For our sample of stars, including many BY Dra-type objects, the correlation between the chromospheric activity, measured by the $\log R_{\mathrm{HK}}^{\prime}$-index and rotation $v \sin i$ and between the Li abundance, rotation, and the $\log R_{\mathrm{HK}}^{\prime}$-index were considered for three groups of the stars with $T_{\text {eff }}$ from 6000 to $5700 \mathrm{~K}, T_{\text {eff }}$ from 5700 to $5200 \mathrm{~K}$ and $T_{\text {eff }}<5200 \mathrm{~K}$. The high Li abundance was detected for a small number of the stars with $T_{\text {eff }}<5600 \mathrm{~K}$. We can make two main conclusions.

1. The correlation Li-activity is evident only in a restricted temperature range ( $T_{\text {eff }}$ from 5700 to $5200 \mathrm{~K}$ ).

2. The Li abundance spread seems to be present in a group of low chromospheric activity stars that also show a broad spread in the chromospheric vs. coronal activity.

Acknowledgements. The authors thank the anonymous referee and Dr. Piercarlo Bonifacio, as referee, for the careful reading of the manuscript and the important remarks that enabled the work to be improved. T.M. thanks the Laboratoire d'Astrophysique de Bordeaux for their kind hospitality during the course of the present project. This study was conducted using the SIMBAD database, operated at the CDS, Strasbourg, France. It is based on the data obtained from the ESA Hipparcos satellite (HIPPARcos catalogue). The present work was supported by the Swiss National Science Foundation, project SCOPES No. IZ73Z0-128180/1. M.K. and M.L. are grateful for the financial support within the framework of RFBR grant 12-02-00884, and RSS grant 2374.2012.2.

\section{Appendix A}

HD 139813 has both high X-ray emission $\left(R_{\mathrm{x}}=-3.75\right)$ and $\log R_{\mathrm{HK}}^{\prime}=-4.40$ (Wright et al. 2004) that indicates it is an active star. Its SIMBAD object type is a star in a double system with high proper motion and IR source.

HD 185414 has $\log R_{\mathrm{HK}}^{\prime}=-4.83$ according to Baliunas et al. (1995). That star is in Takeda et al. (2010) where its measurements indicate moderate activity (HIP 96396, $\left.r_{0}(8542)=0.218\right)$.

HD 208038 is in Wright et al. (2004), but its colour is outside the boundaries for the $\log R_{\mathrm{HK}}^{\prime}$ determination. Its GrandS value (0.533) indicates some Ca II emission, so, it can be considered as an active one.

We noted three stars with $\log R_{\mathrm{HK}}^{\prime}$ in the range -4.55 to -4.70 in Wright et al. (2004), but with no other indication of the activity (HD 4635, HD 105631, HD 184385).

\section{References}

Baliunas, S. L., Donahue, R. A., Soon, W. H., et al. 1995, ApJ, 438, 269 Baranne, A., Queloz, D., Mayor, M., et al. 1996, A\&AS, 119, 373

Barnes, S. A. 2003, ApJ, 586, 464

Benz, W., \& Mayor, M. 1984, A\&A, 138, 183

Canal, R., Isern, J., \& Sanahuja, B. 1975, ApJ, 200, 646

Charbonnel, C., Vauclair, S., \& Zahn, J.-P. 1992, A\&A, 255, 191

Chen, Y. Q., \& Zhao, G. 2006, AJ, 131, 1816

Chen, Y. Q., Nissen, P. E., Benoni, T., \& Zhao, G. 2001, A\&A, 371, 943

Christian, D. J., Mathioudakos, J. D., et al. 2005, A\&A, 632, L1237

Cutispoto, G., Tagliaferri, G., \& de Medeiros, J. R., et al. 2003, A\&A, 397, 987

D’Antona, F., \& Mazzitelli, I. 1984, A\&A, 138, 431

D’Antona, F., \& Mazzitelli, I. 1997, Mem. Soc. Astr. It., 68, 807

Duncan, D. 1981, ApJ, 248, 651

Duncan, D. K., \& Jones, B. F. 1983, ApJ, 271, 663

Favata, F., Micela, G., \& Sciortino, S. 1997, A\&A, 323, 809

Flower, F. 1996, ApJ, 469, 355

Fuhrmann, K. 2008, MNRAS, 384, 173 
Gaidos, E. J., Henry, G. W. \& Henry, S. M. 2000, AJ, 120, 1006

Gaidos, E. J., \& Gonzalez, G. 2002, New A, 7, 211

Galazutdinov, G. A. 1992, Preprint SAO RAS, 92

Girardi, L., Bressan, A., Bertelli, G., \& Chiosi, C. 2000, A\&A, 141, 371

Guedel, M. 2004, A\&ARv, 12, 71

Guinan, E. F., \& Engle, S. G. 2009, The Ages of Stars, Proc. International Astronomical Union, IAU Symp., 258, 395

Hall, J. C., Lockwood, G. W., \& Skiff, B. A. 2007, AJ, 133, 862

Henry, T. J., Soderblom, D. R., Donahue, R. A., \& Baliunas, S. L. 1996, AJ, 111, 439

Herbig, G. H. 1965, ApJ, 141, 588

Huensch, M., Schmitt, J. H. M. M., Sterzik, M. F., \& Voges, W. 1999, A\&ASS, 135,319

Iben, I. Jr. 1965, ApJ, 141, 993

Israelian, G., Santos, N. C., Mayor, M., \& Rebolo, R. 2004, A\&A, 414, 601

Jenkins, J. S., Murgas, F., Rojo, P., et al. 2011, A\&A, 531, A8

Jones, B. F., Fischer, D., Shetrone, M., \& Soderblom, D. R. 1997, AJ, 114, 352

Katsova, M. M., \& Livshits, M. A 2006, Astron. Rep., 50, 579

Katsova, M. M., \& Livshits, M. A. 2011, Astron. Rep., 55, 1123

Katz, D., Soubiran, C., Cayrel, R., et al. 1998, A\&A, 338, 151

King, J. R., Schuler, S. C., Hobbs, L. M., \& Pinsonneault, M. H. 2010, ApJ, 710, 1610

Kovtyukh, V. V., Soubiran, C., \& Belik, S. I. 2004, A\&A, 427, 923

Kurucz, R. L. 1993, CD ROM, 13

Lambert, D. L., Heath, J. E., \& Edvardsson, B. 1991, MNRAS, 253, 610

Lovis, C., Dumusque, X., Santos, N. C., et al. 2011, A\&A, submitted [arXiv: 1107.5325]

Lubin, D., Tytler, D., \& Kirkman, D. 2010, ApJ, 716, 766

Luck, R. E., \& Heiter, U. 2006, AJ, 131, 3069

Livshits, M. A. 1997, Sol. Phys., 173, 377

Maldonado, J., Martínez-Arnáiz, R. M., Eiroa, C., Montes, D., \& Montesinos, B. 2010, A\&A, 521, 12

Mamajek, E. E., \& Hillenbrand, L. A. 2008, ApJ, 687, 1264

Martin, E. L., Basri, G., Pavlenko, Ya., \& Lyubchik, Yu. 2002, ApJ, 579, 437

Martínez-Arnáiz, R., Lóopez-Santiago, J., Crespo-Chacóon, I., \& Montes, D. 2011, MNRAS, 414, 2629

Masana, E., Jordi, C., \& Ribas, I. 2006, A\&A, 450, 735

Michaud, G., Richer, J., \& VandenBerg, D. A. 2004, ApJ, 606, 452

Mishenina, T. V., \& Tsymbal, V. V. 1997, Pis'ma v Astron. Zhurn., 23, 693
Mishenina, T. V., Soubiran, C., Kovtyukh, V. V., \& Korotin, S. A. 2004, A\&A, 418,551

Mishenina, T. V., Soubiran, C., Bienayme, O., et al. 2008, A\&A, 489, 923

Montes, D., \& Ramsey, L. W. 1998 A\&A, 340, L5

Moultaka, J., Ilovaisky, S. A., Prugniel, P., \& Soubiran, C. 2004, PASP, 116, 693

Nordström, B., Mayor, M., Andersen, J., et al. 2004, A\&A, 418, 989

Pallavicini, R., Cerruti-Sola, M., \& Duncan, D. K. 1987, A\&A, 174, 116

Pallavicini, R., Randich, S., \& Giampapa, M. S. 1992, A\&A, 253, 185

Pallavicini, R., Cutispoto, G., Randich, S., \& Gratton, R. 1993, A\&A, 267, 145

Poppenhaeger, K., Robrade, J., \& Schmitt, J. H. M. M. 2010, A\&A, 515, A98

Poppenhaeger, K., Robrade, J., \& Schmitt, J. H. M. M. 2011, A\&A, 529, A1

Queloz, D., Allain, S., Mermilliod, J.-C., Bouvier, J., \& Mayor, M. 1998, A\&A, 335,183

Randich, S. 2010, Proc. IAU Symp., 268, 275

Randich, S., Gratton, R., \& Pallavicini, R. 1993, A\&A, 273, 194

Randich, S., Primas, F., Pasquini, L., Sestito, P., \& Pallavicini, R. 2007, A\&A, 469,163

Schmitt, J. H. M. M., \& Liefke, C. 2004, A\&A, 417, 651

Skumanich, A. 1972, ApJ, 171, 565

Soderblom, D. R., Jones, B. F., Balachandran, S., \& Stauffer, J. R. 1993a, AJ, 106,1059

Soderblom, D. R., Stauffer, J. R., Hudon, J. D., \& Jones, B. F. 1993b, ApJS, 85, 315

Soderblom, D. R., Stauffer, J. R., MacGregor, K. B., \& Jones, B. F. 1993c, ApJ, 409, 624

Takeda, Y., \& Kawanomoto, S. 2005, PASJ, 57, 45

Takeda, Y., Kawanomoto, S., Honda, S., Ando, H., \& Sakurai, T. 2007, A\&A, 468,663

Takeda, Y., Honda, S., Kavanomoto, S., et al. 2010, A\&A, 515, A93

Tatischeff, V., \& Thibaud, J.-P. 2007, A\&A, 469, 265

Thorburn, J. A., Hobbs, L. M., Deliyannis, C. P., \& Pinsonnealt, M. H. 1993, ApJ, 415, 150

Tokovinin, A. A. 1992, A\&A, 256, 121

Tsymbal, V. V. 1996, ASP Conf. Ser., 108, 198

Valenti, J. A., \& Fischer, D. A. 2005, ApJS, 159, 141

Vaughan, A. H., \& Preston, G. W. 1980, PASP, 92, 385

Voges, W., Aschenbach, B., Boller, T., et al. 1999, A\&A, 349, 389

Wright, J. T., Marcy, G. W., Butler, R. P., \& Vogt, S. S. 2004, ApJS, 152, 261 